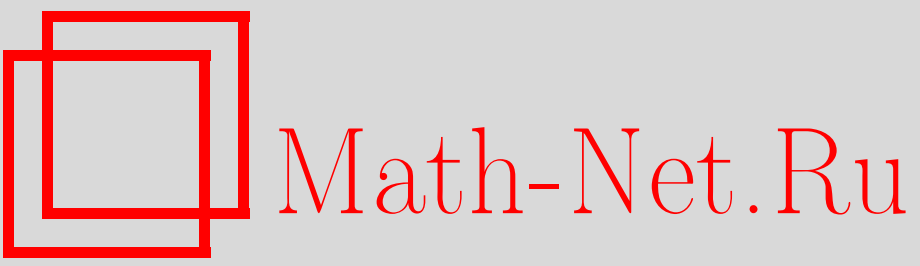

С. А. Мелихов, Псевдогомотопия влечет гомотопию для сингулярных зацеплений коразмерности $\geqslant 3$, УМH, 2000, том 55, выпуск 3, 183-184

DOI: https://doi.org/10.4213/rm303

Использование Общероссийского математического портала Math-Net.Ru подразумевает, что вы прочитали и согласны с пользовательским соглашением

http://www . mathnet.ru/rus/agreement

Параметры загрузки:

IP: 54.196.121.252

26 апреля 2023 г., 06:39:07 


\title{
ПСЕВДОГОМОТОПИЯ ВЛЕЧЕТ ГОМОТОПИЮ ДЛЯ СИНГУЛЯРНЫХ ЗАЦЕПЛЕНИЙ КОРАЗМЕРНОСТИ $\geqslant 3$
}

\author{
С. Мелихов
}

Пусть $X$ и $Q$ - топологические пространства, причем для $X$ фиксировано разложение в несвязное объединение: $X=X_{1} \sqcup \cdots \sqcup X_{k}$. Непрерывное отображение $f: X \rightarrow Q$ называется (обобщенным) сингулярным зачеплением, если $f\left(X_{i}\right) \cap f\left(X_{j}\right)=\varnothing$ при $i \neq j$. Сингулярные зацепления $f_{0}, f_{1}: X \rightarrow Q$ называются $п$ севдогомотопныцми, если существует сингулярное зацепление $F: X \times I=X_{1} \times I \sqcup \cdots \sqcup X_{k} \times I \rightarrow Q \times I$ такое, что $F(x, i)=\left(f_{i}(x), i\right)$ при $i=0,1$ и любом $x \in X$. Если, кроме того, $F$ сохраняет уровни (т.е. если $F^{-1}(Q \times t)=X \times t$ при всех $t \in I$ ), то $f_{0}$ и $f_{1}$ назьваются гомотопным ми. Ниже рассматривается только случай компактного полиэдра $X$ и кусочно-линейного многообразия $Q$; наибольший интерес (см. [1]-[8]) представля-

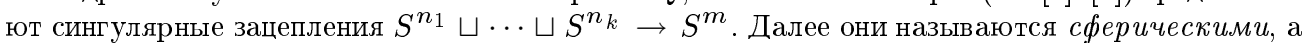
множество их классов эквивалентности по отношению гомотопии обозначается $L M_{n_{1}, \ldots, n_{k}}^{m}$.

В 60-х годах было показано, что в гладкой (Смейл, Хэфлигер, Хадсон) и кусочно-линейной (Зиман, Ликориш, Хадсон) категориях в коразмерности $\geqslant 3$ конкордантные вложения объемлемо изотопны (см. [9]; хорошо известно, что это не так в меньших коразмерностях). Аналогичный вопрос в теории сферических сингулярных зацеплений: влечет ли псевдогомотопия гомотопию в коразмерности $\geqslant 3$ - оставался открытым (см. [4; с. 303], а также [2; замечание 2.4]). Развивая методы Зимана, Ликориша и Хадсона, можно показать, что вопрос решается положительно даже для несколько более общих сингулярных зацеплений, чем сферические:

Теорема 1. Если $m-n \geqslant 3$ и сингулярные зацепления $f_{0}, f_{1}: X \rightarrow Q$ компактного $n$-мерного полиәдра $X=X_{1} \sqcup \cdots \sqcup X_{k}$ в кусочно-линейном $m$-мерном многообразии $Q$ псевдогомотопнь, то они гомотопны.

НАБРОСОК дОКАЗАТЕльСтвА. Пусть $F: X \times I \rightarrow Q \times I$ - данная псевдогомотопия между $f_{0}$ и $f_{1}$. Условимся считать, что "солнце" испускает свои "лучи" в "вертикальном" направлении $I$-слоев $Q \times I$. Допустим, что удалось найти отображение $H: X \times I \rightarrow X \times I$, тождественное на $X \times\{0,1\}$ и такое, что $F$-образ каждого "слоя" $H(X \times t)$ не затеняет сам себя (т.е. никакие две различные точки $F \circ H(X \times t)$ не лежат на одной вертикали). Тогда при замене $F \circ H$ на сохраняющее уровни отображение $G: X \times I \rightarrow Q \times I, G(x, t)=\left(\pi_{Q} \circ F \circ H(x, t), t\right)$, новых двойных точек не появляется. Поскольку $G$ совпадает с $F$ на $X \times\{0,1\}$, это и есть искомая гомотопия.

Чтобы построить $H$, заметим, что без ограничения общности $F$ кусочно-линейно, и по общему положению множество $S$ точек из $X \times I, F$-образы которых лежат на одной вертикали с некоторыми другими, имеет коразмерность $\geqslant 2$ в $X \times I$; в частности, $X_{i} \times I \backslash S$ связно при каждом $i$. Аналогично доказательству леммы Зимана о солнечном сдавливании (см. [9; 5.2]) можно показать, что $X \times I$ сдавливается на $X \times 0$ так, что симплексы $S$ сдавливаются в порядке взаимного затенения их образов и уменьшения размерностей. Небольшой модификацией сдавливания можно добиться того, что на каждом его этапе $F$-образ образа $X \times 1$ при сдавливании не затеняет сам себя. Но тогда отображение $X \times I$ в себя, переводящее точку $(x, t)$ в образ точки $(x, 1)$ в момент $(1-t)$ сдавливания, является требуемым (за исключением того, что оно не тождественно на $X \times 0$, что легко исправить).

Укажем некоторые другие резултаты о соотношении гомотопии и псевдогомотопии; в части коразмерности $\geqslant 3$ все они обобшаются теоремой 1 . Было установлено, что эти два отношения совпадают для сингулярных зацеплений $S^{n_{1}} \sqcup \cdots \sqcup S^{n_{k}} \rightarrow S^{m}$ при $m=3, n_{i}=1$ [5], при $n_{1} \leqslant m-3$ и $n_{2}, \ldots, n_{k} \leqslant \frac{2}{3} m-1$ [3], при $k=2$ и $2 n_{1}+2 n_{2} \leqslant 3 m-5$ [1], а также для сингулярных зацеплений, гомотопных гладко вложеннным, все строгие подзацепления которых гомотопны тривиальным, при $n_{i} \leqslant m-3$ и $n_{2}, \ldots, n_{k} \leqslant k(m-2)-\left(n_{1}+\cdots+n_{k}\right)$ (Нежинский и $[4 ; 8.4 c])$. Тайхнер анонсировал, что гомотопия влечет псевдогомотопию для сферических сингулярных зацеплений коразмерности $\geqslant 2$ [4; сноска на с. 303]. С другой стороны, Саяховой был

Работа выполнена при частичной финансовой поддержке Российского фонда фундаментальных исследований (грант № 99-01-00009). 
получен пример псевдогомотопных, но не гомотопных сингулярных зацеплений $S^{1} \sqcup S^{1} \sqcup S^{2} \rightarrow S^{3}$ [7].

Теорема 1, в частности, утверждает, что при ее условиях любая классификация сингулярных зацеплений по отношению псевдогомотопии (например, классификации Нежинского [6] и Скопенкова [10]) является классификацией и по отношению гомотопии, а любой инвариант гомотопии инвариантен и при псевдогомотопии. Следует отметить, что упомянутые результаты [1], [3], [4] o достаточности псевдогомотопии для гомотопии были получены в качестве следствий полноты и инвариантности при псевдогомотопии некоторых инвариантов гомотопии.

Для любых гомотопических классов $\alpha, \beta \in L M_{n_{1}, \ldots, n_{k}}^{m}$ обычным образом определяется их связная сумма $\alpha \sharp \beta \in L M_{n_{1}}^{m}, \ldots, n_{k}$, причем в коразмерности $\geqslant 3$ (и в некоторых других случаях) это определение корректно (см. [2], [8], ср. с [6]). Отражсение $\rho(\alpha) \in L M_{n_{1}, \ldots, n_{k}}^{m}$ определяется как гомотопический класс сингулярного зацепления $R \circ f \circ r$, где $f: S^{n_{1}} \sqcup \cdots \sqcup S^{n_{k}} \rightarrow S^{m}$ - любой представитель $\alpha$, a $r: S^{n_{1}} \sqcup \cdots \sqcup S^{n_{k}} \rightarrow S^{n_{1}} \sqcup \cdots \sqcup S^{n_{k}}$ и $R: S^{m} \rightarrow S^{m}$ - зеркальные отражения в сферах $S^{n_{i}}$ и $S^{m}$ (действительно, если $f^{\prime}$ - другой представитель $\alpha$, т.е. $f$ и $f^{\prime}$ гомотопны, то $R \circ f \circ r$ и $R \circ f^{\prime} \circ r$ гомотопны “отраженной” гомотопией).

СледСтвиЕ 2. Пусть $n_{i} \leqslant m-3$ при всех $i=1, \ldots, k$. Тогда связная сумма (рассматриваемая в качестве сложения) и отражение (рассматриваемое в качестве обращения) порождают структуру абелевой группь на $L M_{n_{1}, \ldots, n_{k}}^{m}$.

Подобное утверждение было сформулировано Скоттом [8]. Однако, как заметил Кошорке $[2 ; 2.4]$, доказательство этого утверждения содержало пробел: фактически было показано, что отражение - обращение с точностью до псевдогомотопии, а не гомотопии. Пробел закрывает теорема 1; в остальном доказательство следствия аналогично [8] (см. также [2], [6]). Следствие 2 отвечает на $[1 ;$ вопрос 1$]$ в случае коразмерности $\geqslant 3$.

Аналогично теореме 1 доказывается следующая более общая

ТЕОРема 3. Пусть $X^{n}$ - компактный полиәдр, $Q^{m}$ - кусочно-линейное многообразие, $m-n \geqslant 3$, и $F: X \times I \rightarrow Q \times I-$ отображсение такое, что $F^{-1}(Q \times i)=X \times i$ nри $i=0,1$. Для любого $\epsilon>0$ существует сохраняющее уровни отображение $G: X \times I \rightarrow Q \times I$ maкое, что $G(x, i)=F(x, i)$ при $i=0,1$ u всех $x \in X$, и такое, что для любъх $x_{1}, \ldots, x_{l} \in X$ выполнено следующее: $G\left(x_{1} \times I\right) \cap \cdots \cap G\left(x_{l} \times I\right) \neq \varnothing$, только если $F\left(N_{\epsilon}\left(x_{1}\right) \times I\right) \cap \cdots \cap F\left(N_{\epsilon}\left(x_{l}\right) \times I\right) \neq \varnothing$, где $N_{\epsilon}(x)$ обозначает $\epsilon$-окрестность $x \quad в$ некоторой фиксированной метрике.

Из теоремы 3 вытекают, в частности, аналоги теоремы 1 для обобщенных дудлей, т.е. отображений $f: X_{1} \sqcup \cdots \sqcup X_{k} \rightarrow Q$ с $f X_{i} \cap f X_{j} \cap f X_{k}=\varnothing$ при различных $i, j, k$, и для почти вложсений, т.е. отображений $X \rightarrow Q$ с малыми прообразами точек.

Автор выражает благодарность В. М. Нежинскому и А. Б. Скопенкову за введение в круг вопросов, рассматриваемых в данной работе, У. Кошорке за пересылку его статей для ознакомления, П. М. Ахметьеву, Р. Р. Садыкову, К. Р. Салихову, Ю. П. Соловьёву и Е. В. Щепину за полезные обсуждения и ценные замечания.

\section{СПИСОК ЛИТЕРАТУРЫ}

[1] Habegger N., Kaiser U. // Topology. 1998. V. 37. P. 75-94. [2] Koschorke U. // Manuscripta Math. 1988. V. 61. P. 383-415. [3] Koschorke U. // Global Analysis in Modern Mathematics. Houston, TX: Publish or Perish, Inc., 1993. P. 283-299. [4] Koschorke U. // Topology. 1997. V. 36. P. 301-324. [5] Lin X. S. On equivalence relations of links in 3-manifolds. UCSD 1985 (Preprint). [6] Нежинский В. М. I // Алгебра и анализ. 1993. Т. 5. С. 170-190; II // Зап. науч. сем. ПОМИ. 1995. Т. 231. С. 191-196. [7] Саяхова Р. Ф. Сингулярные зацепления типа $(1,1, m)$ в $S^{3}, m>1$ (Препринт). [8] Scott G. P. // Abh. Math. Sem. Univ. Hamburg. 1968. V. 32. P. 186-190. [9] Hudson J. F. P. // Ann. of Math. 1970. V. 91. P. 425-448. [10] Skopenkov A. B. On the generalized Massey-Rolfsen invariant for link maps (Preprint). 